\title{
Light-Activated Signaling in DNA-Encoded Sender-Receiver Architectures
}

\author{
Shuo Yang, Pascal A. Pieters, Alex Joesaar, Bas W. A. Bögels, Rens Brouwers, Iuliia Myrgorodska, \\ Stephen Mann,* and Tom F. A. de Greef*
}

Cite This: ACS Nano 2020, 14, 15992-16002

Read Online

ACCESS | Llll Metrics \& More | 回 Article Recommendations | (s Supporting Information

ABSTRACT: Collective decision making by living cells is facilitated by exchange of diffusible signals where sender cells release a chemical signal that is interpreted by receiver cells. A variety of nonliving artificial cell models have been developed in recent years that mimic various aspects of diffusion-based intercellular communication. However, localized secretion of diffusive signals from individual protocells, which is critical for mimicking biological sender-receiver systems, has remained challenging to control precisely. Here, we engineer lightresponsive, DNA-encoded sender-receiver architectures, where protein-polymer microcapsules act as cell mimics and

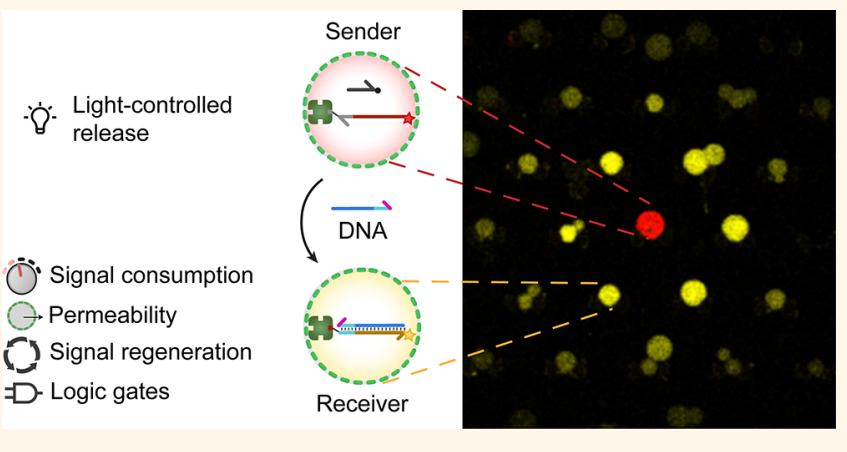
molecular communication occurs through diffusive DNA signals. We prepare spatial distributions of sender and receiver protocells using a microfluidic trapping array and set up a signaling gradient from a single sender cell using light, which activates surrounding receivers through DNA strand displacement. Our systematic analysis reveals how the effective signal range of a single sender is determined by various factors including the density and permeability of receivers, extracellular signal degradation, signal consumption, and catalytic regeneration. In addition, we construct a three-population configuration where two sender cells are embedded in a dense array of receivers that implement Boolean logic and investigate spatial integration of nonidentical input cues. The results offer a means for studying diffusion-based sender-receiver topologies and present a strategy to achieve the congruence of reactiondiffusion and positional information in chemical communication systems that have the potential to reconstitute collective cellular patterns.

KEYWORDS: artificial cells, synthetic biology, DNA strand displacement circuits, molecular communication, microfluidics

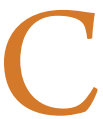

ollective behavior in cellular systems emerges from a tightly choreographed interplay between cellular communication and intracellular signaling processes. ${ }^{1}$ Sender-receiver architectures, where sender cells secrete soluble signals which form a concentration gradient that is interpreted by receiver cells, are ubiquitous in biological systems. ${ }^{2}$ Sender-receiver topologies allow cellular populations to collectively regulate key intracellular events such as cellular growth, ${ }^{3-5}$ cell death, ${ }^{4}$ and cell differentiation ${ }^{6}$ and orchestrate diverse multicellular functions such as tissue regeneration, ${ }^{7}$ cell migration, ${ }^{8}$ immune response amplificatio, $\mathrm{n}^{9-12}$ and robust positioning ${ }^{13-17}$ of cells within a tissue. The effective communication distance over which a single sender can propagate a soluble signal is determined by a number of physicochemical and biological determinants. ${ }^{18}$ Previous work suggests that for many living sender-receiver systems this characteristic length scale is in the order of 50$500 \mu \mathrm{m}^{7,19-21}$ and can be modulated by diverse factors such as cell density, ${ }^{9}$ signal diffusivity, ${ }^{18}$ extracellular signal degrada- tion, $^{22}$ and signal consumption ${ }^{9}$ by receiver cells. However, how each of these factors individually controls the signaling length scale remains unclear.

Synthetic sender-receiver architectures ${ }^{2,23,24}$ based on genetically engineered cells have emerged as excellent tools to establish population-level behaviors such as morphogen reconstitution, ${ }^{17,25}$ artificial networks, ${ }^{26-29}$ Boolean logic gates, ${ }^{30,31}$ and pattern formation, ${ }^{32,33}$ which all arise from the complex interplay of cell-cell communication and intracellular processes. However, quantitative analysis of sender-receiver systems in living cells is complicated by the large number of

Received: September 7, 2020

Accepted: October 14, 2020

Published: October 20, 2020 
A

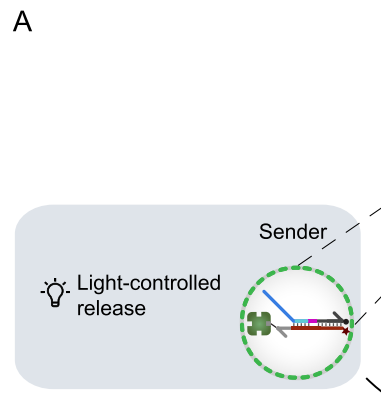

B

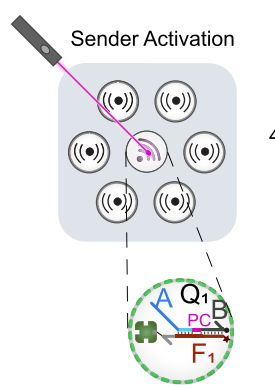

= streptavidin

$A=$ signal

$B=$ fragment with quencher $F_{1}: Q_{1}=$ sender gate complex $\Rightarrow$ Cy5

$\mathrm{PC}=$ photo-cleavable spacer $F_{2}: Q_{2}=$ receiver gate complex

$\longrightarrow$ DNA-based signal

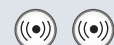
$\underset{\text { laser }}{\stackrel{405 \mathrm{~nm}}{\longrightarrow}}$ $\stackrel{(}{\longrightarrow}$
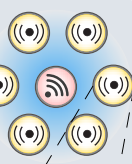

C
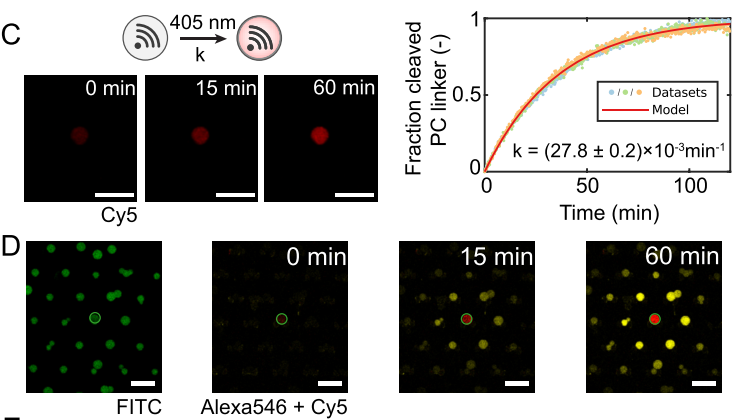

Receiver Activation/ Signal Consumption
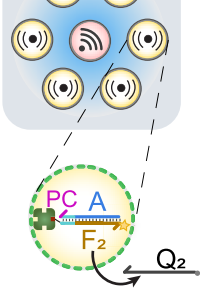

Alexa546 quencher
E
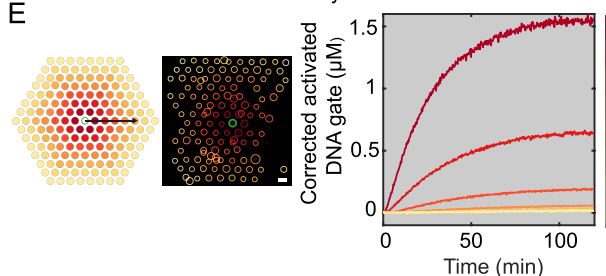

$50 \mu \mathrm{m})$ $2(300 \mu \mathrm{m})$ $3(450 \mu \mathrm{m})$ $4(600 \mu \mathrm{m})$ $5(750 \mu \mathrm{m})$ $6(900 \mu \mathrm{m})$ $7(1050 \mu \mathrm{m})$ $8(1200 \mu \mathrm{m})$ Shells
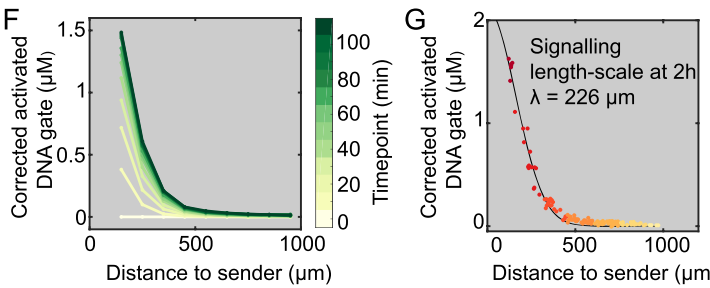

Figure 1. Light-activated DNA-encoded sender-receiver spatial system. (A) A single sender protocell and multiple receiver protocells are localized on a 2D spatial grid using a PDMS-based trapping array. Light-activated release of a ssDNA signal from the sender protocell sets up a signaling gradient which activates nearby receiver protocells. By controlling the characteristics of the protocells and environmentalfactors, this architecture enables quantitative analysis of diffusive signal propagation in space and time and programmable properties. (B) The sender protocell contains a fluorescently quenched internalized gate complex $F_{1} Q_{1}$ anchored to streptavidin using a biotinylated DNA gate strand $\left(F_{1}\right)$. Signal release from the sender protocell is triggered by laser irradiation resulting in photocleavage of strand $Q_{1}$, concomitant dissociation of the two cleaved parts (A and B) and Cy5 fluorescence. Signaling strand A activates the surrounding receiver protocells by displacement of a quencher strand $\left(Q_{2}\right)$ from an internalized streptavidin-anchored gate complex $F_{2} Q_{2}$ to produce an Alexa546 fluorescence output and consumption of strand A. (C) Confocal micrographs of one sender protocell showing time-dependent increase in Cy5 fluorescence upon laser $(405 \mathrm{~nm})$ irradiation, indicating signal release. The plot shows the background-corrected fluorescence trace and exponential fit of the photocleavage reaction with a first-order rate constant of $0.0278 \mathrm{~min}^{-1}$. Experiments were performed in independent triplicates. (D) Confocal micrographs of one sender and multiple receivers (FITC-labeled proteinosome membrane, green) showing timedependent increase in $\mathrm{Cy} 5$ fluorescence (red) and Alexa546 (yellow) fluorescence associated with signal release and activation, respectively. (E) Binning method employed to analyze spatial receiver activation. Protocells are binned in concentric shells based on their distance from the sender (left images). Plots (right) show time-dependent changes in the concentration of activated DNA gates associated with receiver protocells in different concentric shells arranged around the central sender. Shell 1 (dark red) is closest to the sender. The upper limit of the distance from the receiver protocells to the sender protocell of each shell is listed next to the color bars. (F) Concentration of activated DNA gate complexes in receiver protocells plotted for different times as a function of distance to the sender. The fluorescence intensity of each protocell at intervals of exactly $10 \mathrm{~min}$ was obtained through linear interpolation and the average of each distance bin $(50 \mu \mathrm{m}$ per bin) was plotted for these time points (Supporting Information, Supplemental Methods). (G) Plots of concentrations of activated DNA gates in individual receivers positioned at different distances from the sender protocell. Data collected after $2 \mathrm{~h}$ of signal release. The color code corresponds to the different concentric shells as shown in (E). Line represents fit of the data with Gaussian function. Sender protocells and receiver protocells were prepared using 10 and $4 \mu \mathrm{M}$ streptavidin, respectively. Experiments were performed at room temperature. All scale bars are $100 \mu \mathrm{m}$.

variables and context/pathway-specific responses of individual cells. Therefore, a generic platform that would allow quantitative spatiotemporal analysis of sender-receiver architectures remains to be developed. A promising approach to circumvent the use of real cells is to implement synthetic communication modules using protocells which serve as minimalistic model systems for living cells. ${ }^{34-37}$ While current protocell-based systems do not display the rich information processing capabilities of living cells, their minimalistic design allows for rudimentary biological processes to be mimicked with high degrees of control over experimental conditions. Diffusion-mediated communication has been established in bead-based ${ }^{38-41}$ and structurally more cell-like protocell-based systems using a range of soluble signaling factors such as small molecules, ${ }^{42,43}$ proteins, ${ }^{44}$ and DNA. ${ }^{45}$ However, currently no methods exist that can spatially control diffusive signal release at the single protocell level, which is a prerequisite to engineer complex sender-receiver architectures. In addition, while concentration gradients and spatial integration of multiple diffusive signals have been reported for bead-based systems ${ }^{38,46}$ and compartmentalized Belousov-Zhabotinsky (BZ) reactions, ${ }^{47}$ similar advances are less developed for protocells based on biochemical components.

Previously, we have developed the general and scalable platform BIO-PC ${ }^{45}$ (Biomolecular Implementation of Protocellular Communication) capable of distributed interprotocellular molecular communication through DNA strand displacement (DSD) reactions. ${ }^{48}$ Proteinosomes ${ }^{49}$ are used as cell-like 
semipermeable compartments, which contain localized DNA gate complexes and are permeable to short DNA strands that can initiate DSD reactions. Due to the excellent programmability and predictability of DSD, ${ }^{50,51}$ BIO-PC has great potential in mimicking key features of cell-cell communication in living systems. However, spatial control over signal release is absent in the original BIO-PC implementation, which restricts quantitative analysis of sender-receiver systems. In the present work, we adapted the BIO-PC platform for implementation of diffusion-based sender-receiver architectures (Figure 1A). We established sender-receiver systems by sequential localization of a single sender and receiver protocells in a microfluidic trapping array. Spatially controlled signal release from the sender was initiated by light activation resulting in receiver activation by a diffusion-consumption mechanism. ${ }^{9}$ Our work revealed how the corresponding signaling length scale depends on the density and permeability of receivers, signal consumption as a result of receiver activation, and extraprotocellular signal degradation. We then constructed a sender-transceiver system where the diffusible signal responsible for activation of receivers was recycled by a fuel-driven catalytic DSD reaction and revealed an increase in signaling length scale. Finally, we established a spatially encoded Boolean AND gate at the population level where the receiver population integrates nonidentical signals released from two distant senders. Our spatially controlled, DNA-encoded protocell system allows quantitative analysis of diffusionbased sender-receiver architectures and has the potential to uncover design principles of natural cell-cell communication modules.

\section{RESULTS AND DISCUSSION}

Construction of a Light-Activated Spatial DNAEncoded Sender-Receiver System. We adapted the BIOPC platform to function as a sender-receiver architecture by employing two types of streptavidin-containing proteinosomebased semipemeable protocells which were loaded with biotinylated DNA gate complexes capable of sending or receiving short single-stranded DNA strands, respectively (Figure 1B). Our setup is based on preparation of multimodal protocell populations consisting of a single sender and multiple receiver protocells (average protocellular diameter $33.84 \mu \mathrm{m} \pm$ $5.99 \mu \mathrm{m}$, Figure S1) using a microfluidic trapping array. Diffusive molecular communication from the sender to surrounding receiver protocells is initiated by applying laser irradiation to the sender protocell, resulting in cleavage of an internalized photocleavable nitrobenzyl linker and concomitant release of DNA strand A. Strand A functions as the diffusible signal that is secreted from the sender protocell and migrates through the medium, thereby activating surrounding receiver protocells and resulting in a fluorescent response which can be probed with high spatiotemporal resolution (vide infra). Specifically, the sender protocell contains an encapsulated DNA gate complex $F_{1} Q_{1}$ consisting of a fluorophore (Cy5)labeled gate strand $F_{1}$ and strand $Q$ functionalized with a quencher and photocleavable nitrobenzyl moiety (Figure 1B). Upon laser irradiation, strand $Q_{1}$ is cleaved into two shorter ssDNA strands $A$ and $B$ which dissociate from the $F_{1}$ strand at room temperature (Table $\mathrm{S} 1$ ). We characterized the photocleavage of the internalized $\mathrm{F}_{1} \mathrm{Q}_{1}$ gate complex by localizing a single sender protocell in the trapping array followed by irradiation with laser light, resulting in an increase in Cy5 fluorescence of the sender protocell over time due to the cleavage of $\mathrm{Q}$ and dissociation of the quencher-labeled fragment B (Figure 1C and Movie S1). The photocleavage process is observed to follow first order kinetics (Figures 1C and S2). Furthermore, photocleavage of the nitrobenzyl linker inside the sender protocell is localized to the illuminated area and a specific wavelength (Figures S3 and S4). Together, these results validate that the photocleavage of internalized DNA gate complexes inside a sender protocell can be achieved with a high spatial resolution.

Next, we assembled a multimodal sender-receiver population by sequential loading of a single sender and multiple $(\sim 150)$ receiver protocells. The receiver protocells contain an encapsulated DNA gate complex $\mathrm{F}_{2} \mathrm{Q}_{2}$ consisting of a fluorophore (Alexa 546)-labeled gate strand $\mathrm{F}_{2}$ with an exposed toehold domain and a quencher-labeled strand $Q_{2}$ (Figure 1B) resulting in quenching of the Alexa fluorescence. Activation of the receiver protocells is initiated by toeholdmediated strand displacement of $Q_{2}$ by signal strand A released from the sender protocell. Experiments were initiated by laser irradiation ( $405 \mathrm{~nm}$ laser, $2 \mathrm{~h}$ ) of the single sender protocell resulting in photocleavage of the $\mathrm{F}_{1} \mathrm{Q}_{1}$ gate complex which could be monitored by an increase in $\mathrm{Cy} 5$ fluorescence (Figure $1 \mathrm{D}$, red). We confirmed successful activation of the receiver protocells by signal strand A by monitoring an increase in Alexa546 fluorescence in individual receivers (Figure 1D, yellow, and Movie S2). To analyze receiver activation dynamics under the signaling gradient we binned receivers in concentric shells based on their radial distance from the sender and plotted the average fluorescence traces (Figure 1E). Receivers in close proximity to the sender protocell are activated first and to a higher extent, confirming that the diffusible signal released from the central sender is consumed by the surrounding receivers, which therefore limits the activation of receivers at larger distances from the sender. To further quantify the spatiotemporal data, we plotted the spatial distribution of the receiver protocells' activation states at different time points (Figure 1F). The spatiotemporal data displayed an activation front stabilized after 100-120 min. This pseudo steady-state resulted from the diminished signals release from the sender protocell after $2 \mathrm{~h}$ of illumination (Figure 1C). We defined the characteristic signaling length scale $\lambda$ (Supplemental Methods and Figure S5) as the distance at which the receiver activation has dropped off to $1 / e(37 \%)$ of its maximum amplitude at pseudo-steady-state. We determined $\lambda$ from the image taken after $2 \mathrm{~h}$ of illumination and found a characteristic signaling length scale $\lambda$ of approximately $226 \mu \mathrm{m}$ (Figure 1G). This value is well in the range of many natural systems that communicate via soluble factors, i.e., morphogens ${ }^{52}(40-200 \mu \mathrm{m})$, cytokines ${ }^{9}(30-150$ $\mu \mathrm{m})$, and retinoic acid $^{19}(300-500 \mu \mathrm{m})$.

To validate the experimental observations, we performed $2 \mathrm{D}$ reaction-diffusion ( $R D$ ) simulations of the sender-receiver population using the Visual DSD software. ${ }^{45,53,54}$ The numerical model was parametrized using the average signal release rate constant, DSD rate constant, and membrane permeability obtained in separate experiments (Supporting Information, Supplemental Methods). The obtained activation dynamics and signaling length-scale (Figure S6) are in agreement with the experimental results. Collectively, these results establish that spatially controlled light-induced signal release from an individual sender protocell results in a distance-dependent activation of surrounding receivers in agreement with a diffusion-consumption mechanism. 
A Signaling length-scale
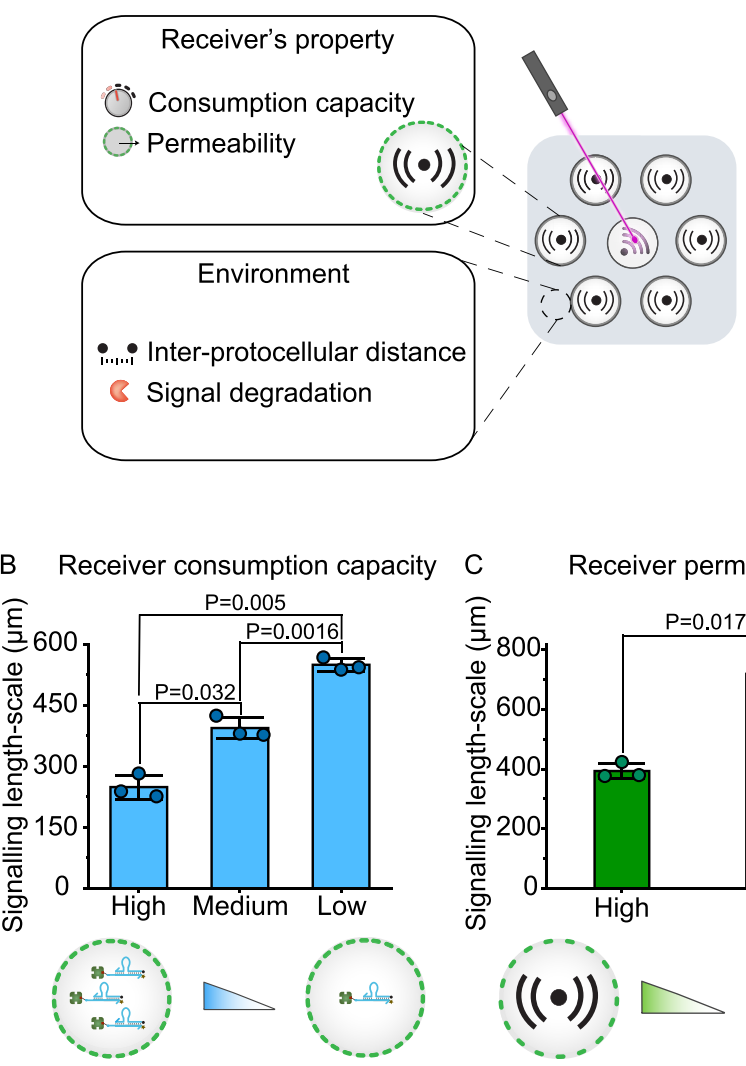

Concentration of DNA gates

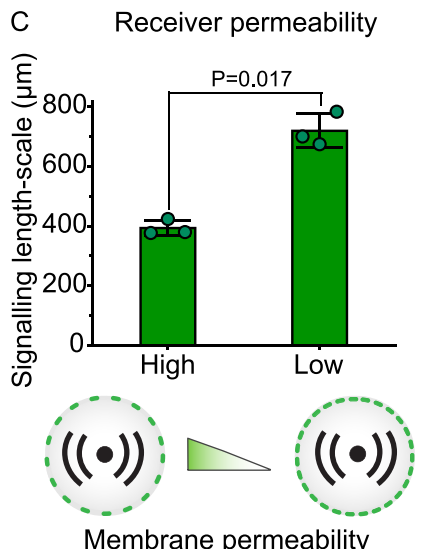

Membrane permeability

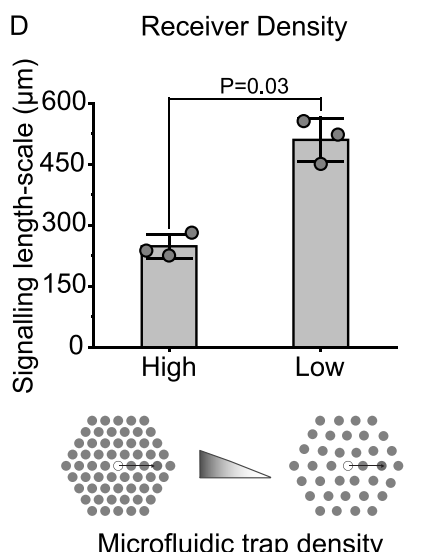

Microfluidic trap density

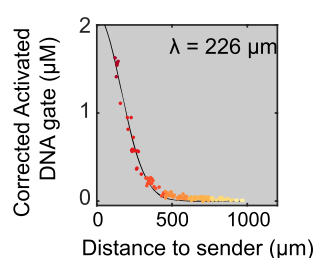

Different

Conditions
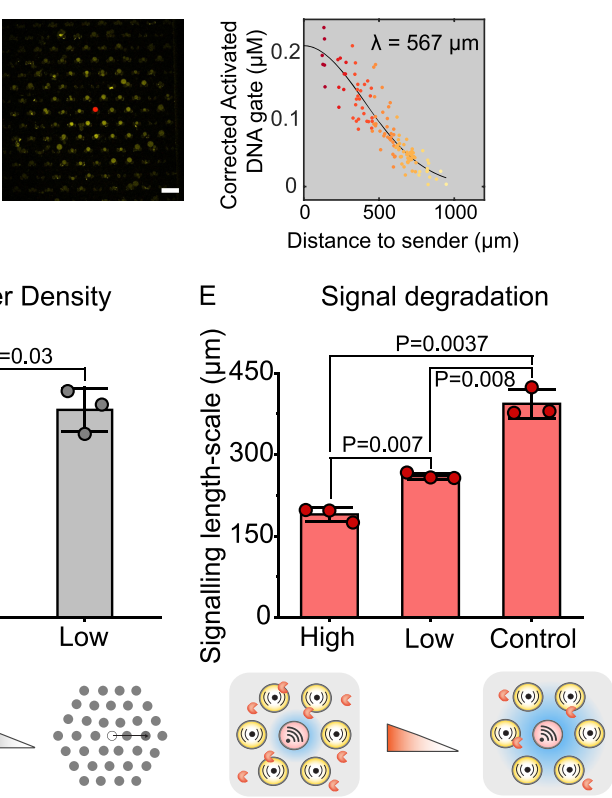

Concentration of exonuclease

Figure 2. Tuning of the signaling length scale in light-activated DNA-encoded sender-receiver spatial systems. (A) Changes in internal factors such as signal consumption capacity (receiver DNA gate complex concentration) and consumption rate (receiver membrane permeability) and external factors such as interprotocellular distance (protocell trap density) and signal degradation (exonuclease concentration) influence the signaling length scale (left and center). Plots show typical experimental data used for the determination of the signaling length scale (right). Data collected after $2 \mathrm{~h}$ of signal release from the sender. Scale bars $150 \mu \mathrm{m}$. (B-E) Modulation of signaling length scale at $t=2 \mathrm{~h}$ for changes in receiver consumption capacity (B), consumption rate (membrane permeability) (C), protocell trap density (D), and signal degradation (E). High, medium, and low levels of the receiver protocell-entrapped DNA gate complex relate to changes in receiver-encapsulated streptavidin concentrations ([SA]) of 4, 1, and $0.4 \mu \mathrm{M}$, respectively $(\mathrm{B})$. High $\left(202.8 \mu \mathrm{m} \mathrm{min}{ }^{-1}\right)$ and low $\left(2.16 \mu \mathrm{m} \mathrm{min}^{-1}\right)$ receiver permeabilities relate to modifications in the protocell membrane cross-linking density; [SA] $=1 \mu \mathrm{M}(\mathrm{C})$. High and low receiver number densities relate to the use of 90 or 70 microfluidic traps per $\mathrm{mm}^{2}$, respectively; $[\mathrm{SA}]=4 \mu \mathrm{M}$ (D). High and low levels of signal degradation arise from the presence of 0.1 and $0.05 \mathrm{unit} / \mu \mathrm{L}$ of exonuclease $\mathrm{I}$, respectively; [SA] $=1 \mu \mathrm{M}$. The control experiment is performed in the absence of exonuclease $I(E)$. For all experiments, the concentration of encapsulated streptavidin in the sender protocells was $10 \mu \mathrm{M}$. All experiments were performed in independent triplicates at room temperature. All the experimental conditions are summarized in Table S2. Data are presented as means \pm SD. A P-value less than 0.05 is considered to be statistically significant.

Modulation of Signaling Range. Intercellular communication distances established by soluble factors in multicellular populations are regulated by both internal and external physicochemical factors, such as signal secretion, diffusion, and consumption rates. ${ }^{11,13}$ How each of these determinants modulates the signaling length scale has remained difficult to analyze due to the intrinsic complexity of natural senderreceiver systems. Here we employ our synthetic senderreceiver architecture and quantitatively analyze the contribution of individual determinants to the signaling length scale. Specifically, we constructed multimodal sender-receiver populations through sequential loading of a single sender and multiple (100-150) receiver protocells. Using this setup, we determined the internal and external determinants leading to changes in effective signaling length scale associated with variations in the capacity and rate of signal consumption, and levels of signal degradation in the environment (Figure 2A). To quantify the influence of the variations, we calculated the signaling length scale from images taken after $2 \mathrm{~h}$ illumination (Supporting Information, Supplemental Methods).

In cellular populations, binding of soluble factors to receptors on neighboring cells results in consumption of the available signal and therefore influences the effective signaling range. ${ }^{1,13,18}$ In the BIO-PC platform, the consumption capacity of individual receiver protocells can be varied by changing the concentration of the encapsulated $\mathrm{F}_{2} \mathrm{Q}_{2}$ DNA gate complex, which depletes the diffusible signal by strand displacement. We performed the sender-receiver experiments using receiver protocells with three different DNA gate complex concentrations (Figure S7) and calculated the corresponding signaling length-scales (Figures 2B, S5, and S8-S15). In general, for the 20-40 $\mu \mathrm{m}$ sized receiver protocells used in this study, the effective signaling length scale ranges between 200 and $500 \mu \mathrm{m}$. In agreement with our expectations, increasing the consumption capacity of individual receiver protocells results in lower effective communication 

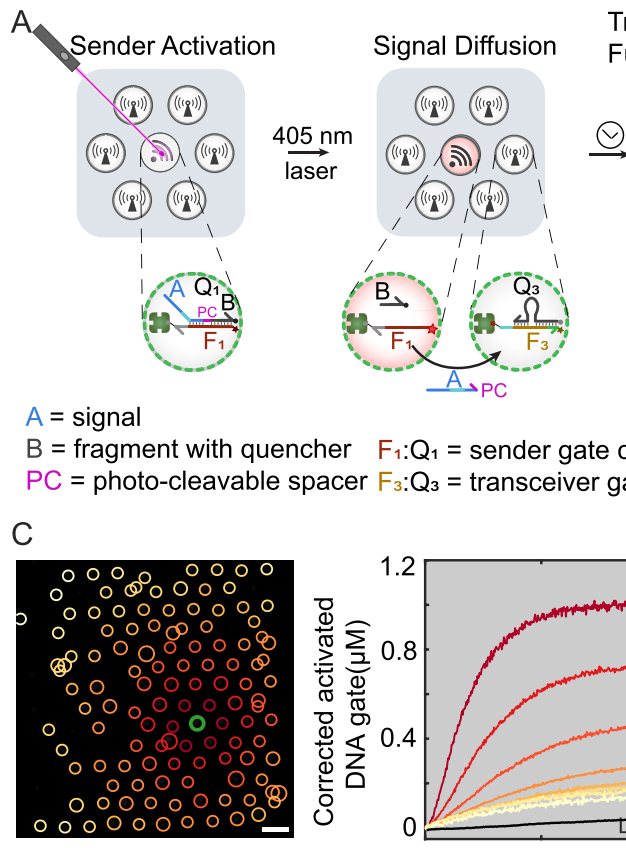

Transceiver Activation/

Fuel-Driven Signal Regeneration

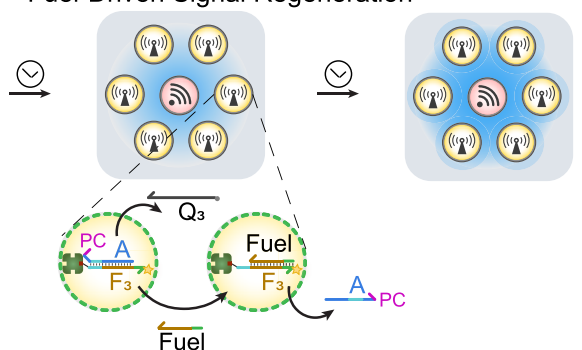

Fuel

$B=$ fragment with quencher $\quad F_{1}: Q_{1}=$ sender gate complex
$P C=$ photo-cleavable spacer $F_{3}: Q_{3}=$ transceiver gate complex $\quad$ Cy5 quencher $\Rightarrow$ Alexa546

$B=$ fragment with quencher $\quad F_{1}: Q_{1}=$ sender gate complex
$P C=$ photo-cleavable spacer $F_{3}: Q_{3}=$ transceiver gate complex $\quad$ Cy5 quencher $*$ Alexa546

$\mathrm{PC}=$ photo-cleavable spacer $\mathrm{F}_{3}: \mathrm{Q}_{3}=$ transceiver gate complex $\cdot$ Cy5 quencher $\cdot$ Alexa546 quencher

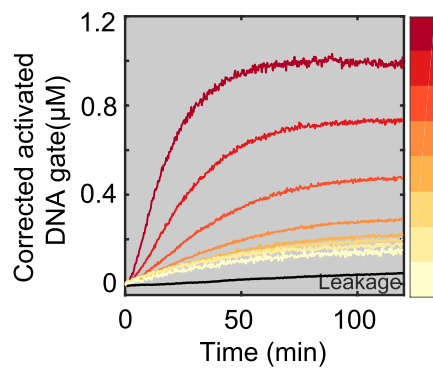

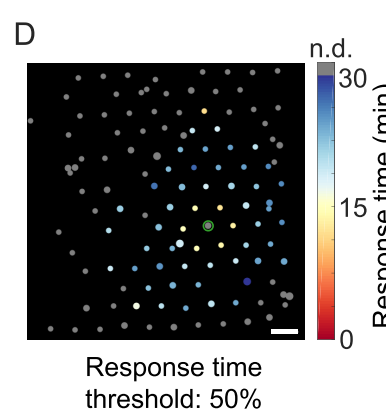

$\mathrm{E}$
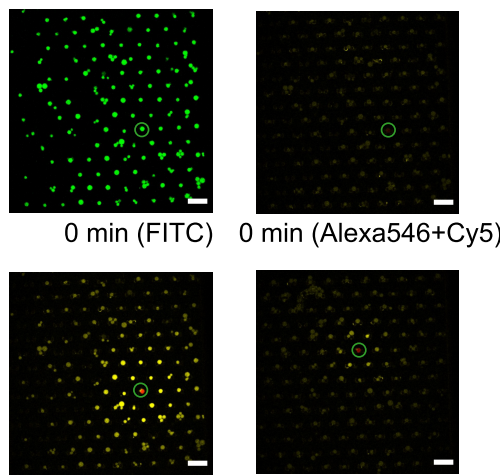

$60 \min$ (no fuel)

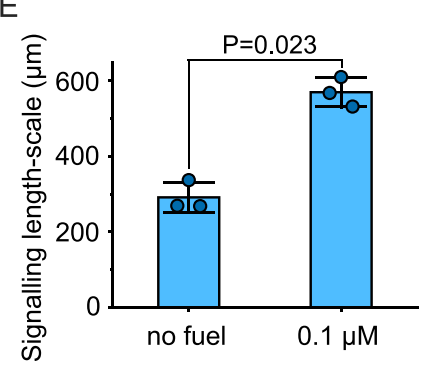

Figure 3. Signal regeneration in a light-activated spatial DNA-encoded sender-transceiver system. (A) The encapsulated sender gate complex is identical to that used for the sender-receiver system. Upon laser irradiation, signal strand A is released to generate Cy5 fluorescence and activates the surrounding transceiver protocells by displacement of a quencher strand $\left(Q_{3}\right)$ from encapsulated gate complex $\mathrm{F}_{3} \mathrm{Q}_{3}$ to produce Alexa546 fluorescence and consumption of strand A. After the initial response, a nonenzymatic DNA catalytic reaction recycles the signal strand A by consuming a fuel strand. (B) Confocal micrographs of one sender and multiple transceivers (FITC-labeled proteinosome membrane, green) recorded at $t=0$ (top left) showing minimal Cy5 and Alexa546 fluorescence before signal activation (top right) in the presence of a fuel strand; corresponding images $60 \mathrm{~min}$ after light-induced signal generation show increases in Cy5 fluorescence in the sender (red, release of signal A) and Alexa546 fluorescence (yellow, activation of $F_{3} Q_{3}$ ) in the surrounding transceivers (bottom left). The control experiment was performed without fuel and shows lower levels of Alexa546 fluorescence after 60 min due to the absence of signal regeneration (bottom right). (C) Binning of transceiver protocell activation (left) and corresponding time traces within different concentric shells for changes in the concentration of transceiver DNA gate activation (right). Shell 1 (dark red) is closest to the sender. The upper limit of the distance from the receiver protocells to the sender protocell of each shell is listed next to the color bars. To analyze spontaneous triggering of the transceiver gates by the fuel, the mean concentration of activated transceiver gate without signal release (i.e., no laser irradiation) is also plotted (black line). (D) Spatial barcode image of response time of transceiver protocells. The response time is defined as the time in which a transceiver reaches $50 \%$ of its final activated concentration; short "on" time (red), long "on" time (blue). To remove background noise, any protocells with an absolute increase less than $20 \mathrm{RFU}$ are excluded and labeled with gray (n.d.). (E) Calculated signaling length-scales in a sender-transceiver system. Data are presented as means \pm SD. A $P$-value less than 0.05 is considered to be statistically significant. Sender and transceiver protocells were prepared using 10 and $1 \mu \mathrm{M}$ streptavidin, respectively. The concentration of fuel was $0.1 \mu \mathrm{M}$. All experiments were performed in independent triplicates at room temperature. Scale bars, $150 \mu \mathrm{m}$.

distances due to a higher local depletion of the soluble signal. Besides consumption capacity, the consumption rate of a soluble signal can also modulate the signaling length scale. In multicellular populations, the consumption rate of a morphogen or cytokine can be regulated by controlling the rate of endocytosis. ${ }^{11}$ Here, we modulate the consumption rate by tuning the membrane permeability of receiver protocells. We have previously shown that the permeability $(\mathrm{P})$ of proteinosomes can be tuned using protein-cross-linking reagents of different length and revealed how the permeability influences the compartmentalized DSD reaction kinetics. ${ }^{45} \mathrm{We}$ prepared high-P and low-P receiver protocells (Supporting Information, Supplemental Methods), quantified their permeability, and confirmed they have approximately similar binding capacity for biotin-labeled DNA (Figure S16). As expected, the experimentally derived signaling length scale is higher for low$\mathrm{P}$ receiver protocells (Figures 2C, S10-S12, and S17-S19) as the soluble signal is consumed at a lower rate.

Because an individual sender is surrounded by multiple receivers, the effective signaling distance not only depends on the consumption rate and consumption capacity of individual receivers but also on the cumulative signal consumption which can be varied by modulating the protocell number density in the spatial array. ${ }^{9}$ We fabricated microfluidic trapping arrays with two different densities of protocell traps and determined the effective signaling range from the experimental data (Figure S20). Our data shows that the signaling length scale increases when protocell density is decreased (Figures 2D, S5, S8, S9, and S21-S23). This increasing communication distance can be explained by lower total signal consumption capacity as the number density of receiver protocell decreases.

Biochemical degradation of diffusible factors is a key regulatory mechanism in morphogenesis and can control both the signaling range and sharpness of the diffusion front. ${ }^{13,14,55}$ To mimic signal degradation, we added exonuclease I $\left(3^{\prime}\right.$ to $\left.5^{\prime}\right)$ to the trapped proteinosomes before initiating the photocleavage reaction. Exonuclease I selectively degrades the diffusible signal from its free $3^{\prime}$ end. Control experiments using proteinosomes containing an encapsulated $3^{\prime}$ fluorescently labeled ssDNA show that exonuclease is 

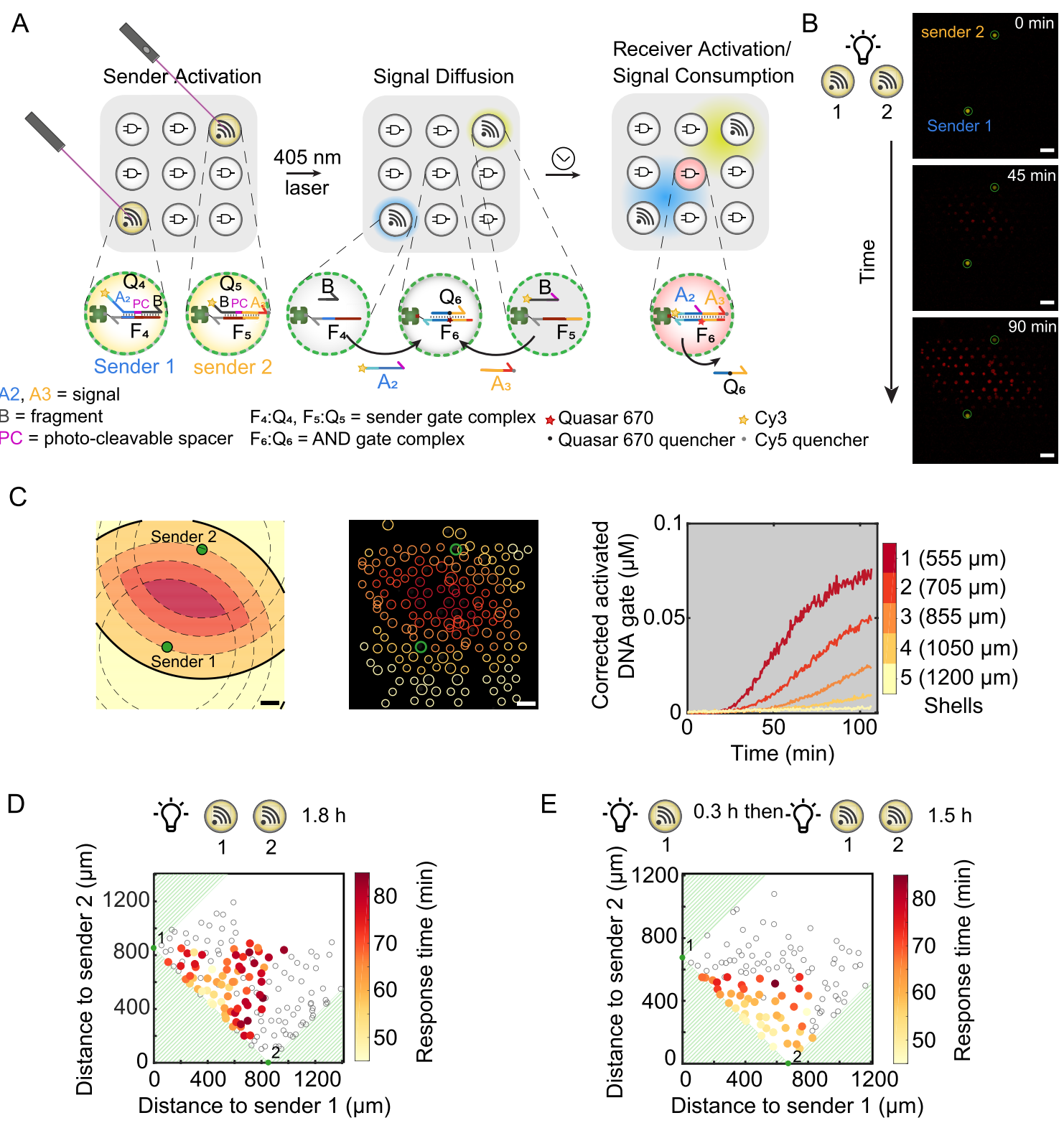

Figure 4. Spatial integration of nonidentical signals by 2D-arrayed AND-gate protocells. (A) Two fluorescent sender protocells (1 and 2) containing internalized gate complexes $F_{4} Q_{4}$ or $F_{5} Q_{5}$, respectively, are embedded in a high number density of nonfluorescent AND-gate receivers. Signal release from sender protocells is triggered by laser irradiation resulting in photocleavage of $Q_{4}$ and $Q_{5}$, concomitant dissociation of the cleaved parts, $A_{2}+B$ and $A_{3}+B$, and loss of $C y 3$ fluorescence. The Cy3-labeled dissociated strand $A_{2}$ and nonfluorescent strand $A_{3}$ activate Quasar 670-quenched receiver protocells containing an encapsulated AND gate $\left(F_{6} Q_{6}\right)$ through cooperative hybridization (64) to produce a Cy3/Quasar670 fluorescence output. (B) Confocal micrographs of two sender protocells (1 and 2) and multiple AND gate receivers recorded at $t=0$ (top) showing $\mathrm{Cy} 3$ fluorescence in the spatially separated transmitters. Light-induced activation leads to a reduction in Cy3 fluorescence (yellow) in the senders, and progressive increase in Quasar 670 fluorescence (red) associated with activation of receiver protocells. (C) Binning method used to analyze spatiotemporal activation of receiver protocells. Protocells are binned based on the maximum of the two distances to the senders, which yields bins with outer bounds that are the intersection of the equivalent bounds of single sender systems, as illustrated by the black lines (left). Corresponding time traces of AND gate receiver activation within different bins. Shell 1 (dark red) is closest to the two senders. The maximum of the two distances from the receiver protocells to the sender protocell of each shell is listed next to the color bars. (D) Response time of individual Boolean receivers upon simultaneous laser irradiation of two senders for $1.8 \mathrm{~h}$ plotted as a function of their distance to each of the two senders. The response time is defined as the time taken for an individual receiver to reach $50 \%$ of its final activated concentration. Two senders are marked as 1 and 2 in green. To remove background noise, any protocells with an absolute increase less than 1 RFU are excluded and labeled in hollow circles. (E) Response time of individual Boolean receivers upon sequential laser irradiation showing spatial bias in activation of receiver protocells. Sender 1 is irradiated for 18 min, followed by irradiation of both senders for $1.5 \mathrm{~h}$. Sender protocells and receiver protocells were prepared using 30 and $1 \mu \mathrm{M}$ streptavidin, respectively. Scale bars are $150 \mu \mathrm{m}$. Experiments were performed at room temperature.

capable of diffusing across the proteinosome membrane (Figure S24) resulting in the presence of exonuclease inside and outside the protocells. However, the encapsulated DNA gate complexes in the sender and receivers lack a free $3^{\prime}$ end, preventing their degradation (Figure S25). Laser-irradiation of the sender protocell cleaves the internalized DNA gate 
complex and yields a diffusible signal strand with a free $3^{\prime}$ end that is amenable for exonuclease degradation. Because the $3^{\prime}$ end of the diffusible signal contains the toehold binding domain required for strand-displacement with the receiver gate complex, enzymatic degradation strongly inhibits receiver activation. We performed sender-receiver experiments for two different concentrations of exonuclease I added to the medium (Figure 2E) and calculated the signaling length scale from the experimental data Figures S10-S12 and S26-S31. As expected, higher concentrations of exonuclease result in decreasing signaling length scales. Taken together, these findings reveal how a fully synthetic sender-receiver protocell platform can be used to systematically study the effect of isolated physicochemical factors on the diffusive communication range.

Signal Regeneration. In living cells, intercellular amplification of soluble signaling molecules is a ubiquitous mechanism employed to direct and control downstream cell fate decisions. ${ }^{56,57}$ Here we implement nonenzymatic DNAbased catalytic reaction networks ${ }^{58}$ and realize interprotocellular signal amplification by engineering the sender-receiver architecture into a sender-transceiver system, where transceiver protocells can be activated by the diffusible signal but are also capable of regenerating this signal in the presence of excess fuel strand (Figure 3A). Similar to the sender-receiver experiments, sender protocells contain encapsulated DNA gate complex $F_{1} Q_{1}$. Transceiver protocells contain encapsulated DNA gate complex $\mathrm{F}_{3} \mathrm{Q}_{3}$ where the streptavidin-bound biotinylated strand $\mathrm{F}_{3}$ is labeled with Alexa546 while strand $\mathrm{Q}_{3}$ is modified with a quencher. Laser irradiation triggers the release of signal A from the sender protocell, which activates the transceiver gate complex by displacing $Q_{3}$. The activated transceiver gate complex $\mathrm{F}_{3} \mathrm{~A}$ exposes the toehold on the $\mathrm{F}_{3}$ strand, allowing an abundantly present fuel strand to bind $F_{3}$ and regenerate signal strand $\mathrm{A}$. We prepared a bimodal protocell population consisting of a single sender and multiple receivers $(\sim 150)$ by sequential loading of protocells in the trapping device. Next, the trapping chamber was filled with fuel strand to a final concentration of $0.1 \mu \mathrm{M}$ and the experiment was initiated by laser irradiation $(405 \mathrm{~nm}, 2 \mathrm{~h})$ of the sender protocell. Comparison of the fluorescent micrographs obtained in the presence and absence of fuel (Figure 3B) clearly reveals transceiver activation at larger distances from the sender in the presence of the fuel, indicative of successful recycling of the diffusing signal. To further characterize sender-transceiver protocell communication, we plotted the transceiver activation dynamics for increasing distances from the sender (Figure 3C) and the response time of each transceiver (Figure 3D). We observed significant higher activation of transceivers at short distances from the sender in the presence of fuel while a significantly higher fraction of transceivers is activated at larger distances from the sender (Figures S32-S37). Importantly, a control experiment in which the individual sender protocell was not irradiated displays low leakage in the presence of fuel (Figure 3C, black line), which is characteristic for catalytic DSD systems. ${ }^{58}$ We also computed the characteristic length scale in the presence and absence of the fuel which reveals a larger signaling range as a result of regeneration of the soluble signal by fuel-driven DSD cycles (Figure 3E). Simulations using a $2 \mathrm{D}$ reaction-diffusion model that incorporates the effect of the fuel-driven signal regeneration confirm this experimental observation (Figure S38). Collectively, these results show that signal regeneration can be integrated into spatial-controlled sender-receiver architectures and leads to an increase in the signaling length scale.

Spatial Integration of Diffusible Signals by Boolean Receivers. Spatial integration of chemical signals by Boolean operations is essential to generate collective behavior in multicellular populations as exemplified by the immune and nervous systems. ${ }^{59,60}$ Although Boolean reaction-diffusion systems have been implemented using the BelousovZhabotinsky reaction, ${ }^{61-65}$ a versatile and tunable platform based on biomolecular reactions is currently lacking. We previously showed the possibilities of implementing Boolean AND logic using BIO-PC, which relied on the sequential hybridization of two different DNA signals in DSD circuitry. ${ }^{45}$ However, this configuration does not allow distinct signal gradients to be integrated spatially, since the AND gate localized in the receiver protocells will sequester one of the signals in the absence of the other. Here, we reveal how the BIO-PC platform can be adapted to integrate nonidentical gradients by localized AND operations based on a cooperative DSD mechanism. Using a sequential loading procedure, we implemented a three-population configuration consisting of two nonidentical sender protocells embedded in a high density of receivers that implement Boolean AND logic (Figure 4A). The two senders contain gate complexes $F_{4} Q_{4}$ and $F_{5} Q_{5}$, respectively, which upon simultaneous laser illumination (405 $\mathrm{nm}, 1.8 \mathrm{~h}$ ) secrete two distinct Cy3-labeled signal strands $\mathrm{A}_{2}$ and $\mathrm{A}_{3}$ as monitored by the decrease in $\mathrm{Cy} 3$ fluorescence of the sender protocells (Figure $4 \mathrm{~B}$ yellow). Receiver protocells contain an encapsulated DNA gate complex which is activated by a cooperative hybridization mechanism ${ }^{66}$ (Figure S39) where both $A_{2}$ and $A_{3}$ need to be simultaneously present to release quencher labeled strand $Q_{6}$, resulting in an increase in Quasar 670 fluorescence (Figure 4B, red). We analyzed spatiotemporal AND-gate receiver activation by binning receiver protocells into shells based on the maximum of the two distances to the senders and calculated the average fluorescence per bin over time (Figure 4C and Figure S40). The experimental curves reveal that activation of the receivers is initiated at positions equidistant to the two senders, in agreement with AND-type logic. Our observations are also supported by $2 \mathrm{D} \mathrm{RD}$ simulations using realistic parameters (Figure S41). Furthermore, we plotted the response time of each receiver as a function of the distance to both senders and find the lowest response time for receivers at equidistant position of both senders (Figure 4D). Together, these results indicate that receiver protocells are activated by two nonidentical signaling gradients of distributed spatial origins and demonstrate Boolean AND logic.

Because receiver protocells are activated by gradients from both senders, we wondered if we could spatially control initiation of receiver activation by sequential laser irradiation of the two senders. This would result in the development of a signal gradient from one of the two senders before the other gradient is established. Due to the reversible nature of the cooperative DSD mechanism, ${ }^{66}$ signal strands from one sender that bind to the AND-gate are preferentially released in the absence of the other signal, preventing signal consumption by receivers. We first irradiated sender 1 for $18 \mathrm{~min}$, followed by exposure of both senders for $1.5 \mathrm{~h}$, and calculated the response time for each receiver (Figures 4E and S42). We observe a skewed activation pattern where receiver activation is initiated in receivers in close proximity to sender 2 , in agreement with the presence of spatial bias in the established signal gradients. 
Together, these results show that a population of protocells could be programmed to integrate two nonidentical cues and perform spatially encoded Boolean operations using encapsulated DSD based reactions.

\section{CONCLUSIONS}

Cellular communication by soluble factors allows populations of cells to coordinate their behavior. Secretion of diffusible messengers is often spatially localized resulting in local gradients near sender cells and the emergence of spatial niches characterized by a high concentration of a specific signal. ${ }^{9}$ For nonmigrating, micrometer-sized cells, the characteristic signaling length scale of these gradients, i.e., the distance over which diffusive communication persists, appears to be around 50$500 \mu \mathrm{m}^{9,19,52}$ and can be dynamically adjusted by competition between diffusion and signal consumption by receiver cells ${ }^{9}$ and active signal degradation. ${ }^{15}$ In this work, we demonstrate a fully synthetic soft matter system based on semipermeable microcompartments that communicate via short DNA strands under a light-induced local signaling gradient arising from a single sender protocell. We prepared multimodal protocell arrays consisting of a single sender protocell and a polydisperse receiver population using a microfluidic trapping device and systematically quantified how individual parameters control the signaling length scale typically between 100 and $700 \mu \mathrm{m}$. The simplicity of the system allows variation of the consumption capacity and consumption rate of receiver protocells and introduction of active signal regeneration and degradation pathways. As a further showcase of our cytomimetic technology, we revealed how two local signaling gradients can be spatially integrated by employing receiver protocells containing Boolean AND gates.

As an artificial communication platform designed for simplicity and tunability, the adapted BIO-PC has its limitations. Living cells can translate extracellular signals into intracellular signals and perform subsequent processing of these signals through highly complex interaction networks of nodes, modules, and pathways. ${ }^{67}$ In contrast, there is no clear distinction between extracellular and intracellular signals in our system, which does not allow coordination of responses via combinatorial signaling. Additional research is needed to construct more complicated DNA-based networks for signal reception and processing. ${ }^{68-71}$ Second, negative feedback control is often employed in multicellular organisms to regulate intercellular communication and guarantees precision, robustness, and versatility. ${ }^{57}$ Because our system is based on enzyme-free DNA circuity, it is difficult to realize negative feedback control. Negative feedback loops can be implemented in BIO-PC using enzyme-assisted DNA circuits, which could be utilized to construct protocell communities with much spatiotemporal behavior. ${ }^{72}$ Moreover, in nature, sender cells consume a fraction of the signal they produced via an autocrine signaling loop. ${ }^{73}$ An artificial autocrine pathway could be created in our system by colocalization of signal and receiver gates inside the protocell or modification of receiver gates onto the protocellular membrane.

For future research, we envision that the BIO-PC platform could form the basis for implementing a deterministic cellular automaton based on chemical reaction-diffusion networks which would be able to perform universal computation and permanently store the chemical state of each protocell. ${ }^{74}$ In order to construct a DNA-based cellular automaton, additional DNA-based Boolean operations such as NOR, XOR, and
NAND gates, which have been shown to work in bulk, ${ }^{70}$ need to be introduced into the protocell platform. Second, the position of each protocell should be independently addressable, which could be achieved using either acoustic ${ }^{75}$ or magnetic ${ }^{76}$ driven manipulation. The development of minimally synthetic cellular communities with programmable communication protocols is a key goal in bottom-up synthetic biology ${ }^{77}$ and has the potential to inform the design rules of collective decision making in multicellular populations.

\section{METHODS}

Streptavidin-containing proteinosomes were prepared similarly to our previously reported procedures. ${ }^{45}$ All DNA sequences are listed in Table S3. DNA oligonucleotides were purchased from Integrated DNA Technologies and Biosearch Technologies. A two-layer PDMS microfluidic chip was produced using standard soft lithographic techniques. $^{78}$ To perform an experiment, protocells were first suspended in the buffer solution and delivered to the trapping chamber in the microfluidic device by a compressed air line. Photocleavage of gate complexes inside a sender protocell was triggered by a $405 \mathrm{~nm}$ laser from a confocal laser scanning microscope (Leica SP8). The confocal microscope was also used to measure fluorescence of protocells in the trap array. Data analysis and 2D reaction-diffusion simulation were performed using Matlab and Vissual DSD. Full details are given in the Supporting Information.

\section{ASSOCIATED CONTENT}

\section{Supporting Information}

The Supporting Information is available free of charge at https://pubs.acs.org/doi/10.1021/acsnano.0c07537.

Experimental, data analysis and simulation methods, Visual DSD code of the simulation, characterization of the protocells (size, permeability, protocellular density), behaviors of the internalized DNA gate complex (concentration determination, signal consumption, degradation, regeneration and Boolean AND logic), photocleavage reaction in the protocells, quantification of the sender-receiver, sender-transceiver, and Boolean AND logic systems in full details, simulated data of the sender-receiver, sender-transceiver, and Boolean AND logic systems, design of the microfluidic device, calculated free energy of the DNA gate complex, summarized tables of the experimental conditions, DNA sequences and simulation parameters, legends for movies (PDF)

Photocleavage reaction inside a sender protocell (AVI)

Photocleavage reaction inside a sender protocell and activation of receiver protocells (AVI)

\section{AUTHOR INFORMATION}

\section{Corresponding Authors}

Stephen Mann - Centre for Protolife Research and Max Planck Bristol Centre for Minimal Biology, School of Chemistry, University of Bristol, Bristol BS8 1TS, United Kingdom; 다이.org/0000-0003-3012-8964; Email: s.mann@ bristol.ac.uk

Tom F. A. de Greef - Laboratory of Chemical Biology, Department of Biomedical Engineering, Computational Biology Group, Department of Biomedical Engineering and Institute for Complex Molecular Systems, Eindhoven University of Technology, Eindhoven 5600 MB, The Netherlands; Institute for Molecules and Materials, Radboud University, Nijmegen 
6525 MB, The Netherlands; 이이. ord.org/0000-0002-9338284X; Email: t.f.a.d.greef@tue.nl

\section{Authors}

Shuo Yang - Laboratory of Chemical Biology, Department of Biomedical Engineering, Computational Biology Group, Department of Biomedical Engineering and Institute for Complex Molecular Systems, Eindhoven University of Technology, Eindhoven 5600 MB, The Netherlands

Pascal A. Pieters - Laboratory of Chemical Biology, Department of Biomedical Engineering, Computational Biology Group, Department of Biomedical Engineering and Institute for Complex Molecular Systems, Eindhoven University of Technology, Eindhoven 5600 MB, The Netherlands; (1) orcid.org/0000-0003-2032-0100

Alex Joesaar - Laboratory of Chemical Biology, Department of Biomedical Engineering, Computational Biology Group, Department of Biomedical Engineering and Institute for Complex Molecular Systems, Eindhoven University of Technology, Eindhoven 5600 MB, The Netherlands

Bas W. A. Bögels - Laboratory of Chemical Biology, Department of Biomedical Engineering, Computational Biology Group, Department of Biomedical Engineering and Institute for Complex Molecular Systems, Eindhoven University of Technology, Eindhoven 5600 MB, The Netherlands

Rens Brouwers - Laboratory of Chemical Biology, Department of Biomedical Engineering, Computational Biology Group, Department of Biomedical Engineering and Institute for Complex Molecular Systems, Eindhoven University of Technology, Eindhoven 5600 MB, The Netherlands

Iuliia Myrgorodska - Centre for Protolife Research and Max Planck Bristol Centre for Minimal Biology, School of Chemistry, University of Bristol, Bristol BS8 1TS, United Kingdom

Complete contact information is available at:

https://pubs.acs.org/10.1021/acsnano.0c07537

\section{Author Contributions}

S.Y. and T.F.A.d.G. designed research; S.Y., A.J., and R.B. performed research; I.M. and S.M. provided key reagents; P.A.P. and A.J. analyzed data; S.Y., P.A.P., A.J., B.W.A.B., R.B., I.M., S.M., and T.F.A.d.G. wrote the paper.

\section{Notes}

The authors declare no competing financial interest.

A preprint of this work has been posted: Yang, S.; Pieters, P. A.; Joesaar, A.; Bögels, B.; Brouwers, R.; Myrgorodska, I.; Mann, S.; de Greef, T. F. A. Light-Activated Signaling in DNAEncoded Sender-Receiver Architectures. BioRxiv, June 17, 2020, ver. 1. DOI: $10.1101 / 2020.06 .10 .144162$.

\section{ACKNOWLEDGMENTS}

We thank P. Li and M. B. Elowitz for sharing Matlab codes used in our data analysis, A. Phillips from Microsoft Research for advice on Visual DSD. S.Y. and T.F.A.d.G. were supported by an NWO-VIDI grant from The Netherlands Organization for Scientific Research (NWO, 723.016.003); P.A.P., A.J., and T.F.A.d.G. were supported by an ERC starting grant by the European Research Council (Project No. 677313 BioCircuit) and Gravity programmes by Ministry of education, culture and Science (024.001.035 and 0.24.003.013); B.W.A.B. was supported by Microsoft Research (PhD Scholarship Programme); I.M. and S.M. were supported by the European Commission (ERC Advanced Grant, 740235).

\section{REFERENCES}

(1) Doğaner, B. A.; Yan, L. K. Q.; Youk, H. Autocrine Signaling and Quorum Sensing: Extreme Ends of a Common Spectrum. Trends Cell Biol. 2016, 26, 262-271.

(2) Barcena Menendez, D.; Senthivel, V. R.; Isalan, M. SenderReceiver Systems and Applying Information Theory for Quantitative Synthetic Biology. Curr. Opin. Biotechnol. 2015, 31, 101-107.

(3) Miller, M. B.; Bassler, B. L. Quorum Sensing in Bacteria. Annu. Rev. Microbiol. 2001, 55, 165-199.

(4) Hart, Y.; Reich-Zeliger, S.; Antebi, Y. E.; Zaretsky, I.; Mayo, A. E.; Alon, U.; Friedman, N. Paradoxical Signaling by a Secreted Molecule Leads to Homeostasis of Cell Levels. Cell 2014, 158, 10221032.

(5) Jayaraman, A.; Wood, T. K. Bacterial Quorum Sensing: Signals, Circuits, and Implications for Biofilms and Disease. Annu. Rev. Biomed. Eng. 2008, 10 (1), 145-167.

(6) Zhu, J.; Yamane, H.; Paul, W. E. Differentiation of Effector CD4 T Cell Populations. Annu. Rev. Immunol. 2010, 28, 445-489.

(7) Chen, C.-C.; Wang, L.; Plikus, M. V.; Jiang, T. X.; Murray, P. J.; Ramos, R.; Guerrero-Juarez, C. F.; Hughes, M. W.; Lee, O. K.; Shi, S.; et al. Organ-Level Quorum Sensing Directs Regeneration in Hair Stem Cell Populations. Cell 2015, 161, 277-290.

(8) Weber, M.; Hauschild, R.; Schwarz, J.; Moussion, C.; De Vries, I.; Legler, D. F.; Luther, S. A.; Bollenbach, T.; Sixt, M. Interstitial Dendritic Cell Guidance by Haptotactic Chemokine Gradients. Science 2013, 339, 328-332.

(9) Oyler-Yaniv, A.; Oyler-Yaniv, J.; Whitlock, B. M.; Liu, Z.; Germain, R. N.; Huse, M.; Altan-Bonnet, G.; Krichevsky, O. A Tunable Diffusion-Consumption Mechanism of Cytokine Propagation Enables Plasticity in Cell-To-Cell Communication in the Immune System. Immunity 2017, 46, 609-620.

(10) Busse, D.; de la Rosa, M.; Hobiger, K.; Thurley, K.; Flossdorf, M.; Scheffold, A.; Höfer, T. Competing Feedback Loops Shape IL-2 Signaling between Helper and Regulatory T Lymphocytes in Cellular Microenvironments. Proc. Natl. Acad. Sci. U. S. A. 2010, 107, 30583063.

(11) Altan-Bonnet, G.; Mukherjee, R. Cytokine-Mediated Communication: A Quantitative Appraisal of Immune Complexity. Nat. Rev. Immunol. 2019, 19, 205-217.

(12) Hodgkin, P. D.; Rush, J.; Gett, A. V.; Bartell, G.; Hasbold, J. The Logic of Intercellular Communication in the Immune System. Immunol. Cell Biol. 1998, 76, 448-453.

(13) Ashe, H. L.; Briscoe, J. The Interpretation of Morphogen Gradients. Development 2006, 133, 385-394.

(14) Green, J. B. A.; Sharpe, J. Positional Information and ReactionDiffusion: Two Big Ideas in Developmental Biology Combine. Development 2015, 142, 1203-1211.

(15) Teleman, A. A.; Cohen, S. M. Dpp Gradient Formation in the Drosophila Wing Imaginal Disc. Cell 2000, 103, 971-980.

(16) Zeng, X.; Goetz, J. A.; Suber, L. M.; Scott, W. J.; Schreiner, C. M.; Robbins, D. J. A Freely Diffusible Form of Sonic Hedgehog Mediates Long-Range Signalling. Nature 2001, 411, 716-720.

(17) Li, P.; Markson, J. S.; Wang, S.; Chen, S.; Vachharajani, V.; Elowitz, M. B. Morphogen Gradient Reconstitution Reveals Hedgehog Pathway Design Principles. Science 2018, 360, 543-548.

(18) Francis, K.; Palsson, B. O. Effective Intercellular Communication Distances Are Determined by the Relative Time Constants for Cyto/Chemokine Secretion and Diffusion. Proc. Natl. Acad. Sci. U. S. A. $1997,94,12258-12262$.

(19) Shimozono, S.; Iimura, T.; Kitaguchi, T.; Higashijima, S.; Miyawaki, A. Visualization of an Endogenous Retinoic Acid Gradient across Embryonic Development. Nature 2013, 496, 363-366.

(20) Martin, C. E.; Spasova, D. S.; Frimpong-Boateng, K.; Kim, H.O.; Lee, M.; Kim, K. S.; Surh, C. D. Interleukin-7 Availability Is Maintained by a Hematopoietic Cytokine Sink Comprising Innate Lymphoid Cells and T Cells. Immunity 2017, 47, 171-182.

(21) Sarris, M.; Masson, J.-B.; Maurin, D.; Van der Aa, L. M.; Boudinot, P.; Lortat-Jacob, H.; Herbomel, P. Inflammatory Chemo- 
kines Direct and Restrict Leukocyte Migration within Live Tissues as Glycan-Bound Gradients. Curr. Biol. 2012, 22, 2375-2382.

(22) Wang, Y.; Wang, X.; Wohland, T.; Sampath, K. Extracellular Interactions and Ligand Degradation Shape the Nodal Morphogen Gradient. eLife 2016, 5, No. e13879.

(23) Toda, S.; Frankel, N. W.; Lim, W. A. Engineering Cell-cell Communication Networks: Programming Multicellular Behaviors. Curr. Opin. Chem. Biol. 2019, 52, 31-38.

(24) Pai, A.; Tanouchi, Y.; Collins, C. H.; You, L. Engineering Multicellular Systems by Cell-cell Communication. Curr. Opin. Biotechnol. 2009, 20, 461-470.

(25) Carvalho, A.; Menendez, D. B.; Senthivel, V. R.; Zimmermann, T.; Diambra, L.; Isalan, M. Genetically Encoded Sender-Receiver System in 3D Mammalian Cell Culture. ACS Synth. Biol. 2014, 3, 264-272.

(26) Bacchus, W.; Lang, M.; El-Baba, M. D.; Weber, W.; Stelling, J.; Fussenegger, M. Synthetic Two-Way Communication between Mammalian Cells. Nat. Biotechnol. 2012, 30, 991-996.

(27) Basu, S.; Mehreja, R.; Thiberge, S.; Chen, M.-T.; Weiss, R. Spatiotemporal Control of Gene Expression with Pulse-Generating Networks. Proc. Natl. Acad. Sci. U. S. A. 2004, 101, 6355-6360.

(28) Balagaddé, F. K.; Song, H.; Ozaki, J.; Collins, C. H.; Barnet, M.; Arnold, F. H.; Quake, S. R.; You, L. A Synthetic Escherichia Coli Predator-Prey Ecosystem. Mol. Syst. Biol. 2008, 4, 187.

(29) Youk, H.; Lim, W. A. Secreting and Sensing the Same Molecule Allows Cells to Achieve Versatile Social Behaviors. Science 2014, 343, 1242782.

(30) Regot, S.; Macia, J.; Conde, N.; Furukawa, K.; Kjellén, J.; Peeters, T.; Hohmann, S.; De Nadal, E.; Posas, F.; Solé, R. Distributed Biological Computation with Multicellular Engineered Networks. Nature 2011, 469, 207-211.

(31) Tamsir, A.; Tabor, J. J.; Voigt, C. A. Robust Multicellular Computing Using Genetically Encoded NOR Gates and Chemical 'Wires.'. Nature 2011, 469, 212-215.

(32) Basu, S.; Gerchman, Y.; Collins, C. H.; Arnold, F. H.; Weiss, R. A Synthetic Multicellular System for Programmed Pattern Formation. Nature 2005, 434, 1130-1134.

(33) Ortiz, M. E.; Endy, D. Engineered Cell-Cell Communication via DNA Messaging. J. Biol. Eng. 2012, 6, 16.

(34) Villar, G.; Graham, A. D.; Bayley, H. A Tissue-Like Printed Material. Science 2013, 340, 48-52.

(35) Salehi-Reyhani, A.; Ces, O.; Elani, Y. Artificial Cell Mimics as Simplified Models for the Study of Cell Biology. Exp. Biol. Med. 2017, 242, 1309-1317.

(36) Aufinger, L.; Simmel, F. C. Establishing Communication between Artificial Cells. Chem. - Eur. J. 2019, 25, 12659-12670.

(37) Booth, M. J.; Schild, V. R.; Graham, A. D.; Olof, S. N.; Bayley, H. Light-Activated Communication in Synthetic Tissues. Sci. Adv. 2016, 2, No. e1600056.

(38) Isalan, M.; Lemerle, C.; Serrano, L. Engineering Gene Networks to Emulate Drosophila Embryonic Pattern Formation. PLoS Biol. 2005, 3, e64.

(39) Gines, G.; Zadorin, A. S.; Galas, J.-C.; Fujii, T.; Estevez-Torres, A.; Rondelez, Y. Microscopic Agents Programmed by DNA Circuits. Nat. Nanotechnol. 2017, 12, 351-359.

(40) Frezza, B. M.; Cockroft, S. L.; Ghadiri, M. R. Modular MultiLevel Circuits from Immobilized DNA-Based Logic Gates. J. Am. Chem. Soc. 2007, 129, 14875-14879.

(41) Yashin, R.; Rudchenko, S.; Stojanovic, M. N. Networking Particles over Distance Using Oligonucleotide-Based Devices. J. Am. Chem. Soc. 2007, 129, 15581-15584.

(42) Tang, T. Y. D.; Cecchi, D.; Fracasso, G.; Accardi, D.; CoutablePennarun, A.; Mansy, S. S.; Perriman, A. W.; Anderson, J. L. R.; Mann, S. Gene-Mediated Chemical Communication in Synthetic Protocell Communities. ACS Synth. Biol. 2018, 7, 339-346.

(43) Adamala, K. P.; Martin-Alarcon, D. A.; Guthrie-Honea, K. R.; Boyden, E. S. Engineering Genetic Circuit Interactions within and between Synthetic Minimal Cells. Nat. Chem. 2017, 9, 431.
(44) Niederholtmeyer, H.; Chaggan, C.; Devaraj, N. K. Communication and Quorum Sensing in Non-Living Mimics of Eukaryotic Cells. Nat. Commun. 2018, 9, 5027.

(45) Joesaar, A.; Yang, S.; Bögels, B.; van der Linden, A.; Pieters, P.; Kumar, B. P.; Dalchau, N.; Phillips, A.; Mann, S.; de Greef, T. F. A. DNA-Based Communication in Populations of Synthetic Protocells. Nat. Nanotechnol. 2019, 14, 369-378.

(46) Zadorin, A. S.; Rondelez, Y.; Gines, G.; Dilhas, V.; Urtel, G.; Zambrano, A.; Galas, J.-C.; Estévez-Torres, A. Synthesis and Materialization of a Reaction-Diffusion French Flag Pattern. Nat. Chem. 2017, 9, 990.

(47) Toiya, M.; Vanag, V. K.; Epstein, I. R. Diffusively Coupled Chemical Oscillators in a Microfluidic Assembly. Angew. Chem. 2008, 120, 7867-7869.

(48) Zhang, D. Y.; Winfree, E. Control of DNA Strand Displacement Kinetics Using Toehold Exchange. J. Am. Chem. Soc. 2009, 131, 17303-17314.

(49) Huang, X.; Li, M.; Green, D. C.; Williams, D. S.; Patil, A. J.; Mann, S. Interfacial Assembly of Protein-Polymer Nano-Conjugates into Stimulus-Responsive Biomimetic Protocells. Nat. Commun. 2013, 4, 2239.

(50) Simmel, F. C.; Yurke, B.; Singh, H. R. Principles and Applications of Nucleic Acid Strand Displacement Reactions. Chem. Rev. 2019, 119, 6326-6369.

(51) Zhou, X.; Yao, D.; Hua, W.; Huang, N.; Chen, X.; Li, L.; He, M.; Zhang, Y.; Guo, Y.; Xiao, S.; et al. Programming Colloidal Bonding Using DNA Strand-Displacement Circuitry. Proc. Natl. Acad. Sci. Proc. Natl. Acad. Sci. U. S. A. 2020, 117, 5617-5623.

(52) Müller, P.; Schier, A. F. Extracellular Movement of Signaling Molecules. Dev. Cell 2011, 21, 145-158.

(53) Lakin, M. R.; Youssef, S.; Polo, F.; Emmott, S.; Phillips, A. Visual DSD: A Design and Analysis Tool for DNA Strand Displacement Systems. Bioinformatics 2011, 27, 3211-3213.

(54) Dalchau, N.; Seelig, G.; Phillips, A. Computational Design of Reaction-Diffusion Patterns Using DNA-Based Chemical Reaction Networks. In DNA Computing and Molecular Programming; Springer: Kyoto, 2014; pp 84-99.

(55) Briscoe, J.; Small, S. Morphogen Rules: Design Principles of Gradient-Mediated Embryo Patterning. Development 2015, 142, 3996-4009.

(56) Murphy, K.; Weaver, C. Janeway's Immunobiology, Ninth ed.; Garland science/Taylor and Francis: New York, 2016; pp 1-36.

(57) Freeman, M. Feedback Control of Intercellular Signalling in Development. Nature 2000, 408, 313-319.

(58) Zhang, D. Y.; Turberfield, A. J.; Yurke, B.; Winfree, E. Engineering Entropy-Driven Reactions and Networks Catalyzed by DNA. Science 2007, 318, 1121-1125.

(59) Gidon, A.; Zolnik, T. A.; Fidzinski, P.; Bolduan, F.; Papoutsi, A.; Poirazi, P.; Holtkamp, M.; Vida, I.; Larkum, M. E. Dendritic Action Potentials and Computation in Human Layer 2/3 Cortical Neurons. Science 2020, 367, 83-87.

(60) Altan-Bonnet, G.; Mora, T.; Walczak, A. M. Quantitative Immunology for Physicists. Phys. Rep. 2020, 849, 1-83.

(61) Parrilla-Gutierrez, J. M.; Sharma, A.; Tsuda, S.; Cooper, G. J. T.; Aragon-Camarasa, G.; Donkers, K.; Cronin, L. A Programmable Chemical Computer with Memory and Pattern Recognition. Nat. Commun. 2020, 11, 1442.

(62) Taylor, A. F.; Tinsley, M. R.; Wang, F.; Huang, Z.; Showalter, K. Dynamical Quorum Sensing and Synchronization in Large Populations of Chemical Oscillators. Science 2009, 323, 614-617.

(63) Adamatzky, A.; De Lacy Costello, B. Experimental Logical Gates in a Reaction-Diffusion Medium: The XOR Gate and Beyond. Phys. Rev. E: Stat. Phys., Plasmas, Fluids, Relat. Interdiscip. Top. 2002, 66, 46112.

(64) Tóth, A.; Showalter, K. Logic Gates in Excitable Media. J. Chem. Phys. 1995, 103, 2058-2066.

(65) Tompkins, N.; Li, N.; Girabawe, C.; Heymann, M.; Ermentrout, G. B.; Epstein, I. R.; Fraden, S. Testing Turing's Theory 
of Morphogenesis in Chemical Cells. Proc. Natl. Acad. Sci. U. S. A. 2014, 111, 4397-4402.

(66) Zhang, D. Y. Cooperative Hybridization of Oligonucleotides. J. Am. Chem. Soc. 2011, 133, 1077-1086.

(67) Papin, J. A.; Hunter, T.; Palsson, B. O.; Subramaniam, S. Reconstruction of Cellular Signalling Networks and Analysis of Their Properties. Nat. Rev. Mol. Cell Biol. 2005, 6, 99-111.

(68) Chen, Y.-J.; Dalchau, N.; Srinivas, N.; Phillips, A.; Cardelli, L.; Soloveichik, D.; Seelig, G. Programmable Chemical Controllers Made from DNA. Nat. Nanotechnol. 2013, 8, 755-762.

(69) Seelig, G.; Soloveichik, D.; Zhang, D. Y.; Winfree, E. EnzymeFree Nucleic Acid Logic Circuits. Science 2006, 314, 1585-1588.

(70) Qian, L.; Winfree, E.; Bruck, J. Neural Network Computation with DNA Strand Displacement Cascades. Nature 2011, 475 (7356), 368-372.

(71) Qian, L.; Winfree, E. Scaling up Digital Circuit Computation with DNA Strand Displacement Cascades. Science 2011, 332, 11961201.

(72) Padirac, A.; Fujii, T.; Rondelez, Y. Bottom-up Construction of in Vitro Switchable Memories. Proc. Natl. Acad. Sci. U. S. A. 2012, 109, E3212-E3220.

(73) Berg, H. C.; Purcell, E. M. Physics of Chemoreception. Biophys. J. 1977, 20, 193-219.

(74) Scalise, D.; Schulman, R. Emulating Cellular Automata in Chemical Reaction-Diffusion Networks. Nat. Comput. 2016, 15, 197214.

(75) Tian, L.; Li, M.; Patil, A. J.; Drinkwater, B. W.; Mann, S. Artificial Morphogen-Mediated Differentiation in Synthetic Protocells. Nat. Commun. 2019, 10, 3321.

(76) Li, Q.; Li, S.; Zhang, X.; Xu, W.; Han, X. Programmed Magnetic Manipulation of Vesicles into Spatially Coded Prototissue Architectures Arrays. Nat. Commun. 2020, 11, 232.

(77) Schwille, P. Bottom-up Synthetic Biology: Engineering in a Tinkerer's World. Science 2011, 333, 1252-1254.

(78) Unger, M. A.; Chou, H.-P.; Thorsen, T.; Scherer, A.; Quake, S. R. Monolithic Microfabricated Valves and Pumps by Multilayer Soft Lithography. Science 2000, 288, 113-116. 